International Electronic Journal of Algebra

Volume 27 (2020) 218-236

DOI: $10.24330 /$ ieja.663075

\title{
INDECOMPOSABLE NON UNISERIAL MODULES OF LENGTH THREE
}

\author{
Gabriella D'Este \\ Received: 2 May 2019; Revised: 8 October 2019; Accepted: 21 October 2019 \\ Communicated by Christian Lomp
}

\begin{abstract}
We investigate a particular class of indecomposable modules of length three, defined over a $K$-algebra, with a simple socle and two non isomorphic simple factor modules. These modules may have any projective dimension different from zero. On the other hand their composition factors may have any countable dimension as vector spaces over the underlying field $K$. Moreover their endomorphism rings are $K$-vector spaces of dimension $\leq 2$.
\end{abstract}

Mathematics Subject Classification (2010): 16G20, 16D99

Keywords: Pseudo projective module, quasi projective module, quiver and representation

\section{Introduction}

The aim of this note is to construct left $R$-modules $M$, where $R$ is a $K$-algebra over some field $K$, with the following properties:

(1) $M$ has exactly three non zero proper submodules $N_{1}, N_{2}$ and $N_{1} \cap N_{2}$.

(2) $M / N_{1}$ is not isomorphic to $M / N_{2}$.

(3) End $M / N_{i}$ is isomorphic to $K$ for $i=1,2$.

Given a module $M$, we say that $M$ is quasi projective (resp. pseudo projective) if, for any submodule $X$ of $M$, any morphism (resp. any epimorphism) $f: M \rightarrow M / X$ is of the form $\pi \circ g$, where $\pi: M \rightarrow M / X$ is the canonical epimorphism and $g$ is an endomorphism of $M$. By [4, Lemma 2.1] any module $M$ satisfying (1) and (2) is not quasi projective. Moreover, if (1) and (2) holds, then $M$ is pseudo projective if and only if it satisfies (3) with $K=\mathbb{Z}_{2}$. In the sequel we shall say, for short, that $M$ satisfies (123) if $M$ satisfies conditions (1), (2) and (3). If $x$ is a vertex of a quiver $Q$, and $R$ is the $K$-algebra given by $Q$, then $x$ also denotes the simple module $\left(V_{i}, f_{j}\right)$, with $V_{x}=K, V_{i}=0$ for $i \neq x$ and $f_{j}=0$ for any $j$. Moreover, $P(x)$ and $I(x)$ denote the projective and injective module corresponding to the vertex $x$. In the examples constructed in [4] $R$ is always a $K$-algebra given by quivers with at least 2 vertices and at most 4 vertices, and the modules satisfying (123) are three dimensional 
vector spaces. In this note we consider algebras given by quivers with $n$ vertices for any $n \geq 1$. By making one point extensions or coextensions, or by changing the relations, we obtain modules satisfying (123) of any injective dimension (Proposition 2.1 and of any projective dimension $\geq 1$ (Proposition 2.2. We also show that modules satisfying (123) are very far from being selforthogonal (Examples $2.3,2.4$ and 2.5. and that they cannot be faithfully balanced (Proposition 3.8. Moreover their endomorphism ring is a rather small commutative algebra (Theorem 3.6. As we shall see, the free algebra $K\langle x, y\rangle$ admits modules of dimension three satisfying (123) (Example 2.5, Proposition 2.6. Corollaries 3.10 and 3.11). On the other hand, the free algebra $K<x, y, z\rangle$ admits modules satisfying (123) of any countable dimension $\geq 3$ (Theorem 3.2 . Finally, suitable matrix algebras admit modules satisfying (123) of any infinite dimension over $K$ (Proposition 3.4 and Corollary 3.5.

This paper is organized as follows. In Section 1 we recall some definitions, we fix some conventions and we describe the main results. In Section 2 we investigate homological dimensions and extensions of modules satisfying (123). Finally, in Section 3 , we describe composition factors and endomorphism rings of these modules. For more background on generalizations of projective modules we refer to [3] and [5. For more background on quivers and their representations we refer to [1] and [2].

\section{Homological dimensions and extensions}

We begin with two results on homological dimensions.

Proposition 2.1. Any $d \in \mathbb{N} \cup\{\infty\}$ is the injective dimension of a module $M$ satisfying (123).

Proof. If $d=0,1, \infty$, then the assertion follows from [4, Example 2.3 and Theorem 2.8]. Assume now $2 \leq d<\infty$. Let $n=d+3$ and let $R$ be the $K$-algebra given by the 
following quiver with relations $a b=0$ for all arrows $a$ and $b$.

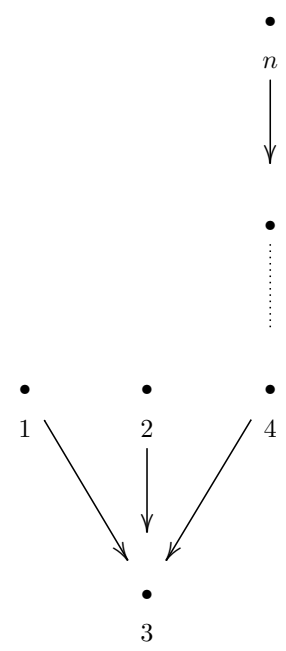

Next let $M$ be the module $\begin{gathered}12 \\ 3\end{gathered}$. Then the injective coresolution of $M$ is

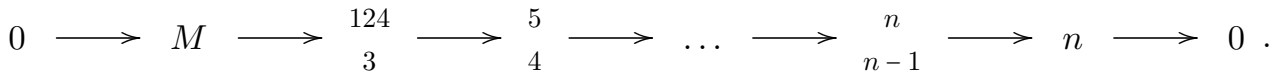

Hence $n-3=d$ is the injective dimension of $M$.

Proposition 2.2. Any $d$ with $1 \leq d \leq \infty$ is the projective dimension of a module $M$ satisfying (123).

Proof. The modules $\begin{gathered}12 \\ 3\end{gathered}$ and $\begin{gathered}12 \\ 2\end{gathered}$ in [4, Examples 2.3 and 2.4] have projective dimension 1 and $\infty$, respectively. Assume now $1<d<\infty$ and let $n=d+2$. Next let $R$ be the $K$-algebra given by the following quiver with relations $a b=0$ for all 
arrows $a$ and $b$.
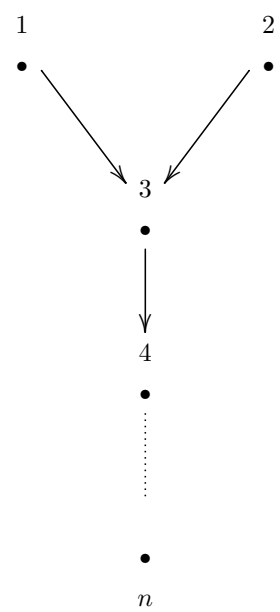

Let $M$ be the module $I(3)=\begin{gathered}12 \\ 3\end{gathered}$. Then the projective resolution of $M$ is

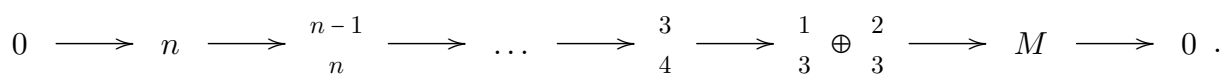

Consequently the projective dimension of $M$ is $n-2=d$.

Propositions 2.1 and 2.2 suggest that we investigate the groups of selfextensions of the modules satisfying (123).

Example 2.3. There is a module $M$ satisfying (123) such that $\operatorname{Ext}^{n}(M, M) \simeq K$ for any $n \geq 1$.

Construction. As in 4, Theorem 2.8], let $R$ be the $K$-algebra given by the quiver $\longrightarrow \stackrel{a}{\longrightarrow} \cdot{ }_{2}$ with relations $b a=0$ and $b^{3}=0$. Next let $M$ be the module $\begin{gathered}12 \\ 2\end{gathered}$. Then $I(1)=1$ and $I(2)=\begin{array}{r}2 \\ 12\end{array}$ are the indecomposable injective modules. From the exact sequence $0 \rightarrow M \stackrel{2}{\rightarrow} I(2) \rightarrow 2 \rightarrow 0$ we obtain the long exact sequence $0 \longrightarrow \operatorname{Hom}(M, M) \stackrel{\simeq}{\longrightarrow} \operatorname{Hom}(M, I(2)) \stackrel{0}{\longrightarrow} \operatorname{Hom}(M, 2) \stackrel{\simeq}{\longrightarrow} \operatorname{Ext}^{1}(M, M) \longrightarrow 0 \longrightarrow$ $\ldots \longrightarrow 0 \longrightarrow \operatorname{Ext}^{i}(M, 2) \longrightarrow \operatorname{Ext}^{i+1}(M, M) \longrightarrow 0 \ldots \ldots$

Consequently we have

$$
\operatorname{Ext}^{1}(M, M) \simeq \operatorname{Hom}(M, 2) \simeq K
$$

and

$$
\operatorname{Ext}^{n+1}(M, M) \simeq \operatorname{Ext}^{n}(M, 2) \quad \text { for any } \quad n \geq 1
$$


On the other hand, from the exact sequence $0 \longrightarrow 2 \longrightarrow I(2) \longrightarrow 1 \oplus{ }_{2}^{2} \longrightarrow$ 0 , we obtain the long exact sequence $0 \longrightarrow \operatorname{Hom}(M, 2) \longrightarrow \operatorname{Hom}(M, I(2)) \longrightarrow$ $\operatorname{Hom}\left(M, 1 \oplus \begin{array}{l}2 \\ 2\end{array}\right) \longrightarrow \operatorname{Ext}^{1}(M, 2) \longrightarrow 0 \ldots \longrightarrow 0 \longrightarrow \operatorname{Ext}^{i}\left(M, 1 \oplus \begin{array}{l}2 \\ 2\end{array}\right) \longrightarrow$ $\operatorname{Ext}^{i+1}(M, 2) \longrightarrow 0 \longrightarrow \ldots$

Hence, comparing dimensions and using the fact that 1 is an injective module, we have

$$
\operatorname{Ext}^{1}(M, 2) \simeq K
$$

and

$$
\operatorname{Ext}^{n+1}(M, 2) \simeq \operatorname{Ext}^{n}\left(M, \begin{array}{l}
2 \\
2
\end{array}\right) \quad \text { for any } \quad n \geq 1 .
$$

Combining (2) and (3), we get

$$
\operatorname{Ext}^{2}(M, M) \simeq K
$$

Finally, from the exact sequence $0 \longrightarrow{ }_{2}^{2} \longrightarrow I(2) \longrightarrow 1 \oplus 2 \longrightarrow 0$, we obtain the long exact sequence $0 \longrightarrow \operatorname{Hom}\left(M, \begin{array}{l}2 \\ 2\end{array}\right) \longrightarrow \operatorname{Hom}(M, I(2)) \longrightarrow \operatorname{Hom}(M, 1 \oplus$ $2) \longrightarrow \operatorname{Ext}^{1}\left(M, \begin{array}{l}2 \\ 2\end{array}\right) \longrightarrow 0 \longrightarrow \ldots \longrightarrow 0 \longrightarrow \operatorname{Ext}^{i}(M, 1 \oplus 2) \longrightarrow \operatorname{Ext}^{i+1}\left(M, \begin{array}{l}2 \\ 2\end{array}\right) \longrightarrow$ $0 \rightarrow \ldots$ Therefore we have

$$
\operatorname{Ext}^{1}\left(M, \begin{array}{l}
2 \\
2
\end{array}\right) \simeq K
$$

and

$$
\operatorname{Ext}^{n+1}\left(M, \begin{array}{l}
2 \\
2
\end{array}\right) \simeq \operatorname{Ext}^{n}(M, 2) \quad \text { for any } \quad n \geq 1 .
$$

Putting (2), (4) and (6) together, we get

$$
\operatorname{Ext}^{3}(M, M) \simeq K
$$

Combining (4) and (7), we obtain

$$
\operatorname{Ext}^{i+2}(M, 2) \simeq \operatorname{Ext}^{i}(M, 2) \quad \text { for any } \quad i \geq 1 .
$$

This result and (2) imply that

$$
\operatorname{Ext}^{i+3}(M, M) \simeq \operatorname{Ext}^{i+1}(M, M) \quad \text { for any } \quad i \geq 1 .
$$

Putting (1), (5), (8) and (10) together, we conclude that $\operatorname{Ext}^{n}(M, M) \simeq K$ for any $n \geq 1$.

As the next examples show, we may have $\operatorname{Ext}^{1}(M, M) \neq 0$ also when $M$ is defined over hereditary algebras. 
Example 2.4. There is a module $M$ satisfying (123) such that $\operatorname{dim} \operatorname{Ext}^{1}(M, M)=$ 2.

Construction. Let $R$ be the infinite dimensional $K$-algebra given by the quiver ${ }_{a} \bigcirc_{1} \stackrel{b}{\longrightarrow} \cdot$. Let $M$ be the module described by the following picture

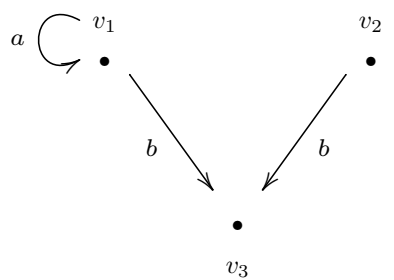

where $\left\{v_{1}, v_{2}, v_{3}\right\}$ is a basis of $M$ and $a v_{1}=v_{1}, a v_{2}=0, b v_{1}=v_{3}=b v_{2}$. Then $R v_{1}, R v_{2}$ and $R v_{1} \cap R v_{2}=R v_{3}$ are the three non zero proper submodules of $M$. On the other hand the three composition factors of $M$ are one dimensional. Let $U_{1}=M / R v_{1}$ an let $U_{2}=M / R v_{2}$. Then $a U_{1}=0$ and $a U_{2} \neq 0$. Hence $M$ satisfies (123). From the exact sequence

$$
0 \longrightarrow R v_{3} \simeq P(2) \longrightarrow M \longrightarrow U_{1} \oplus U_{2} \longrightarrow 0
$$

we get the long exact sequence

$$
\begin{aligned}
0=\operatorname{Hom}\left(U_{1} \oplus U_{2}, M\right) & \longrightarrow \operatorname{Hom}(M, M) \stackrel{\simeq}{\longrightarrow} \operatorname{Hom}\left(R v_{3}, M\right) \stackrel{0}{\longrightarrow} \\
& \longrightarrow \operatorname{Ext}^{1}\left(U_{1} \oplus U_{2}, M\right) \stackrel{\simeq}{\longrightarrow} \operatorname{Ext}^{1}(M, M) \longrightarrow 0 .
\end{aligned}
$$

Let $e_{1}$ be the path of length zero around the vertex 1 . Then the definition of $U_{1}$ and $U_{2}$ guarantees the existence of epimorphisms $f: P(1) \longrightarrow U_{1}$ and $g: P(1) \longrightarrow U_{2}$ such that $\operatorname{Ker} f=R a \oplus R b$ and $\operatorname{Ker} g=R\left(e_{1}-a\right) \oplus R b$. Consequently, there are exact sequences of the form

$$
0 \longrightarrow P(1) \oplus P(2) \longrightarrow P(1) \longrightarrow U_{i} \longrightarrow 0 \quad \text { for } \quad i=1,2 \text {. }
$$

Hence also the following long sequences are exact

$$
\begin{aligned}
0=\operatorname{Hom}\left(U_{i}, M\right) \longrightarrow \operatorname{Hom}(P(1), M) & \longrightarrow \operatorname{Hom}(P(1) \oplus P(2), M) \longrightarrow \\
& \longrightarrow \operatorname{Ext}^{1}\left(U_{i}, M\right) \longrightarrow 0 \quad \text { for } i=1,2 .
\end{aligned}
$$

Since $\operatorname{Hom}(P(1), M) \simeq K^{2}$ and $\operatorname{Hom}(P(1) \oplus P(2), M) \simeq K^{3}$, we deduce from (4) that $\operatorname{Ext}^{1}\left(U_{i}, M\right) \simeq K$ for $i=1,2$. Consequently we deduce from (2) that $\operatorname{dim} \operatorname{Ext}^{1}(M, M)=2$.

In the next example we use a quiver with one vertex. 
Example 2.5. The free algebra $A=K\langle x, y\rangle$ admits a module $M$ satisfying (123) such that $\operatorname{dim} \operatorname{Ext}^{1}(M, M)=10$.

Construction. Let $M$ be the $A$-module described by the following picture

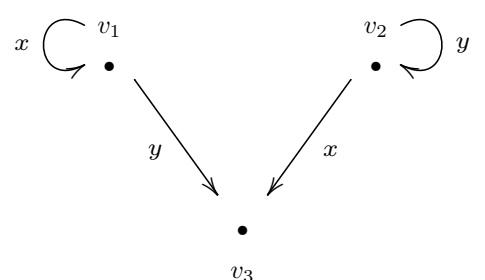

More precisely, let $\left\{v_{1}, v_{2}, v_{3}\right\}$ be a basis of $M$ and assume that $x v_{1}=v_{1}, y v_{2}=v_{2}$, $y v_{1}=v_{3}=x v_{2}$ and $x v_{3}=0=y v_{3}$. Also in this case $A v_{1}, A v_{2}$ and $A v_{1} \cap A v_{2}=A v_{3}$ are the three non zero proper submodules of $M$. Moreover the three composition factors $S_{1}=A v_{1} / A v_{3}, S_{2}=A v_{2} / A v_{3}$ and $S_{3}=A v_{3}$ are pairwise non isomorphic. Hence $M$ satisfies (123). From the exact sequence

$$
0 \longrightarrow A^{2} \longrightarrow A \longrightarrow S_{i} \longrightarrow 0 \quad \text { for } \quad i=1,2,3,
$$

we obtain the long exact sequences

$$
\begin{aligned}
0 \longrightarrow \operatorname{Hom}\left(S_{i}, M\right) \longrightarrow \operatorname{Hom}(A, M) & \longrightarrow \operatorname{Hom}\left(A^{2}, M\right) \longrightarrow \\
& \longrightarrow \operatorname{Ext}^{1}\left(S_{i}, M\right) \longrightarrow 0 \quad \text { for any } i .
\end{aligned}
$$

Since $\operatorname{Hom}\left(S_{i}, M\right)=0$ for $i=1,2$ and $\operatorname{Hom}\left(S_{3}, M\right) \simeq K$, we obtain

$$
\operatorname{dim} \operatorname{Ext}^{1}\left(S_{i}, M\right)=3 \quad \text { for } \quad i=1,2
$$

and

$$
\operatorname{dim} \operatorname{Ext}^{1}\left(S_{3}, M\right)=4 \text {. }
$$

Since the sequence $0 \longrightarrow S_{3} \longrightarrow M \longrightarrow S_{1} \oplus S_{2} \longrightarrow 0$ is exact and $A$ is hereditary, we obtain the long exact sequence

$$
\begin{aligned}
& 0=\operatorname{Hom}\left(S_{1} \oplus S_{2}, M\right) \longrightarrow \operatorname{Hom}(M, M) \stackrel{\simeq}{\longrightarrow} \operatorname{Hom}\left(S_{3}, M\right) \longrightarrow \\
& \longrightarrow \operatorname{Ext}^{1}\left(S_{1} \oplus S_{2}, M\right) \longrightarrow \operatorname{Ext}^{1}(M, M) \longrightarrow \operatorname{Ext}^{1}\left(S_{3}, M\right) \longrightarrow 0 .
\end{aligned}
$$

Putting (2), (3) and (4) together, we conclude that $\operatorname{dim}_{\operatorname{Ext}^{1}}(M, M)=10$.

In the next statement we compare the dimensions of certain subspaces of small modules satisfying (123).

Proposition 2.6. Let $A$ be the free $K$-algebra $K\langle x, y\rangle$, and let $M$ be a module satisfying (123) with $\operatorname{dim} M=3$. Then, among others, the following cases are possible: 
(i) $\operatorname{dim} x M=\operatorname{dim} y M=2$;

(ii) $\operatorname{dim} x M=\operatorname{dim} y M=1$;

(iii) $\operatorname{dim} x M=2, \operatorname{dim} y M=1$.

Proof. (i) This follows from Example 2.5. where we have $x M=\left\langle v_{1}, v_{3}\right\rangle$ and $y M=\left\langle v_{2}, v_{3}\right\rangle$.

(ii) Let $M$ be the $A$-module, obtained in an obvious way from Example 2.4 with basis $\left\{v_{1}, v_{2}, v_{3}\right\}$ described by the following picture.

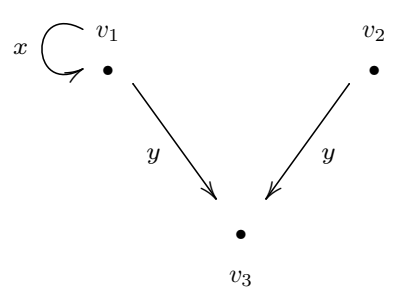

Then we have $x M=\left\langle v_{1}\right\rangle$ and $y M=\left\langle v_{3}\right\rangle$.

(iii) Let $M$ be the $A$-module with basis $\left\{v_{1}, v_{2}, v_{3}\right\}$ described by the following picture.

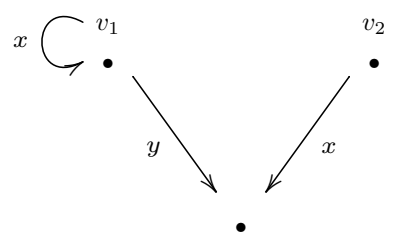

$v_{3}$

then we have $x M=\left\langle v_{1}, v_{3}\right\rangle$ and $y M=\left\langle v_{3}\right\rangle$.

\section{Composition factors and endomorphism rings}

In the sequel we denote by $\mathbb{N}^{+}$the set of positive integers. In order to construct modules satisfying (123) of infinite dimension, we need the following lemma.

Lemma 3.1. Let $A=K\langle x, y\rangle$. Then for any $d \in \mathbb{N}^{+} \cup\left\{\aleph_{0}\right\}$ there is a simple module $S$ with $\operatorname{dim} S=d$ and End $S \simeq K$.

Proof. If $d=1$, the assertion is obvious. Assume $d$ is finite and $d>1$. Let $S$ be the module with basis $\left\{v_{1}, \ldots, v_{d}\right\}$ described in an obvious way by the following picture.

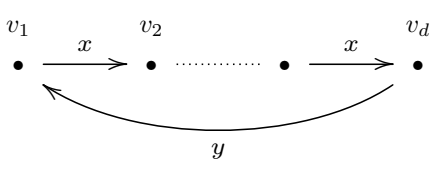


If $0 \neq v=\sum_{i=1}^{d} k_{i} v_{i}$, then we have $y x^{i} v=k_{d-i} v_{1}$ for $i=0, \ldots, d-1$. Consequently $A v=A v_{1}=S$. Hence $S$ is a simple module of dimension $d$. Assume now $f \in \operatorname{End} S$ and $f\left(v_{1}\right)=\sum_{i=1}^{d} k_{i} v_{i}$. Since $y x^{i} v_{1}=0$ for $i=0, \ldots, d-2$, we have $k_{d-i} v_{1}=y x^{i} f\left(v_{1}\right)=$ $f\left(y x^{i} v_{1}\right)=0$ for $i=0, \ldots, d-2$. Hence $k_{d}=k_{d-1}=\cdots=k_{2}=0$, and so $f$ is the multiplication by $k_{1}$. Assume $d=\aleph_{0}$. Let $S$ be the module with basis $\left\{v_{n} \mid n \geq 1\right\}$ described in obvious way by the following picture.

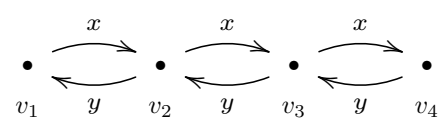

If $0 \neq v=\sum_{i=1}^{n} k_{i} v_{i}$ with $k_{n} \neq 0$, then we have $y^{n-1} v=k_{n} v_{1}$. Consequently $A v=$ $A v_{1}=S$, and so $S$ is a simple module of dimension $\aleph_{0}$. Assume now $f \in \operatorname{End} S$ and $f\left(v_{1}\right)=\sum_{i=1}^{n} k_{i} v_{i}$ for some $n \geq 2$. Since $y v_{1}=0$, it follows that $\sum_{i=1}^{n-1} k_{i+1} v_{i}=$ $y f\left(v_{1}\right)=f\left(y v_{1}\right)=0$. Hence we have $k_{2}=\cdots=k_{n}=0$, and so $f$ is the multiplication by $k_{1}$. The lemma is proved.

By dealing with reasonably large free $K$-algebras it is easy to construct big modules satisfying (123).

Theorem 3.2. If $d_{i} \in \mathbb{N}^{+} \cup\left\{\aleph_{0}\right\}$ for $i=1,2,3$ and $A=K\langle x, y, z\rangle$, then there is an $A$-module $M$, satisfying (123), such that the three composition factors of $M$ have dimensions $d_{1}, d_{2}, d_{3}$ and $d_{3}=\operatorname{dim} \operatorname{soc} M$.

Proof. Let $M$ be the $A$-module with basis $B_{1} \cup B_{2} \cup B_{3}$ with $\left|B_{i}\right|=d_{i}$ for any $i$ described by the following picture.

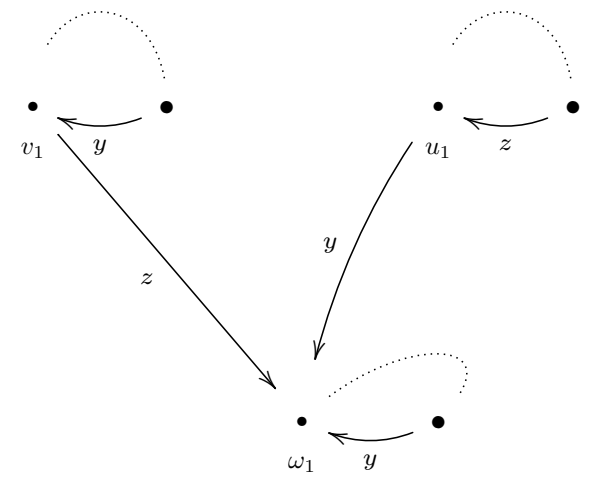

More precisely we have $B_{1}=\left\{v_{i}\right\}_{i \geq 1}, B_{2}=\left\{u_{i}\right\}_{i \geq 1}, B_{3}=\left\{\omega_{i}\right\}_{i \geq 1}$ and the following conditions hold. If $d_{1}=1$, then $y v_{1}=v_{1}, x v_{1}=0$ and $z v_{1}=\omega_{1}$. If $d_{1}>1$, then $B_{1}$ generates the $K\langle x, y\rangle$-module of dimension $d_{1}$ constructed in Lemma 3.1 and we have $z v_{1}=\omega_{1}$ and $z v_{i}=0$ for any $i>1$. If $d_{2}=1$, then $z u_{1}=u_{1}, x u_{1}=0$ and $y u_{1}=\omega_{1}$. If $d_{2}>1$, then $B_{2}$ generates the $K\langle x, z\rangle$-module of dimension $d_{2}$ (with $z$ 
instead of $y$ ) constructed in Lemma 3.1, and we have $y u_{1}=\omega_{1}$ and $y u_{i}=0$ for any $i>1$. If $d_{3}=1$, then $y \omega_{1}=\omega_{1}$ and $x \omega_{1}=0=z \omega_{1}$. If $d_{3}>1$, then $B_{3}$ generates the $K\langle x, y\rangle$-module of dimension $d_{3}$ constructed in Lemma 3.1, and we have $z \omega_{i}=0$ for any $i$. Let now $N_{1}=A v_{1}, N_{2}=A u_{1}$. Then we have $N_{1} \cap N_{2}=A \omega_{1}$, and so $\operatorname{dim} N_{1} \cap N_{2}=d_{3}$. On the other hand $\operatorname{dim} M / N_{1}=d_{2}$ and $\operatorname{dim} M / N_{2}=d_{1}$. Moreover, by Lemma 3.1, $K$ is the endomorphism ring of $M / N_{1}, M / N_{2}$ and $N_{1} \cap N_{2}$. To end the proof, take any $0 \neq m \in M$. If $m \in N_{1} \cap N_{2}$, then we have $A m=A \omega_{1}=N_{1} \cap N_{2}$. If $m \notin N_{1}$, then there exists $a \in A$ and $k \in K$ such that $z a m=u_{1}+k \omega_{1}$. Consequently there is some $b \in A$ such that $z b\left(u_{1}+k \omega_{1}\right)=u_{1}$, and so $N_{2}=A u_{1} \subseteq A m$. Suppose now $m \notin N_{2}$. Then there exist $a \in A$ and $s \in N_{1} \cap N_{2}$ such that yam $=v_{1}+s$. Consequently $\omega_{1}=z y a m \in A m$, and so $N_{1} \cap N_{2}=A \omega_{1} \subseteq A m$. It follows that $v_{1}=$ yam $-s \in A m$; hence $N_{1}=A v_{1} \subseteq A m$. Putting things together, we conclude that $A m=N_{1}$ if $m \in N_{1} \backslash N_{2}, A m=N_{2}$ if $m \in N_{2} \backslash N_{1}$, and $A m=N_{1}+N_{2}=M$ if $m \notin N_{1} \cup N_{2}$. Hence $M$ satisfies (123) and the proof is complete.

Corollary 3.3. Let $R$ be the $K$-algebra given by the quiver

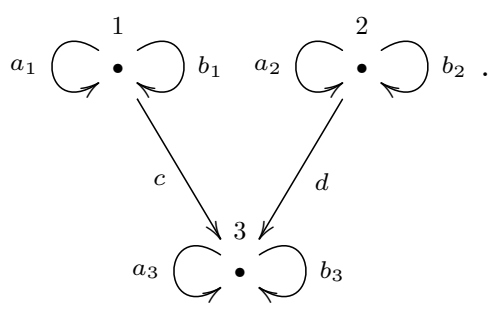

If $d_{i} \in \mathbb{N}^{+} \cup\left\{\aleph_{0}\right\}$ for $i=1,2,3$, then there is an $R$-module $V=\left(V_{i}, f_{i}\right)$ such that $V$ satisfies (123) and $\operatorname{dim} V_{i}=d_{i}$ for any $i$.

Proof. By Lemma 3.1 we can define a simple $K\langle x, y\rangle$-module $\left(V_{i} ; a_{i}, b_{i}\right)$ with dimension $d_{i}$ and endomorphism $\operatorname{ring} K$ for any $i=1,2,3$. Next we fix a non zero map $c: V_{1} \longrightarrow V_{3}$ and a non zero map $d: V_{2} \longrightarrow V_{3}$. In this way we obtain an $R$-module $V=\left(V_{1}, V_{2}, V_{3} ; f_{j}\right)$ with the following properties:

- The three non zero proper submodules of $V$ are $N_{1}=\left(V_{1}, 0, V_{3} ; f_{j}\right), N_{2}=$ $\left(0, V_{2}, V_{3} ; f_{j}\right)$ and $N_{1} \cap N_{2}=\left(0,0, V_{3} ; f_{j}\right)$.

- $V / N_{1}$ is not isomorphic to $V / N_{2}$.

- End $V / N_{i}$ is isomorphic to $K$ for $i=1,2$.

Consequently $V$ satisfies (123) and $\operatorname{dim} V_{i}=d_{i}$ for any $i$.

By hypothesis, two non isomorphic composition factors belonging to the top of a module satisfying (123) have the smallest possible endomorphism ring. However 
it is easy to see that the socle of a module satisfying (123) may have a large endomorphism ring.

Proposition 3.4. Let $E$ be an extension field of $K$ with $[E: K]>1$. Then there are a $K$-algebra $R$ and a faithful $R$-module $M$, satisfying (123), such that $\operatorname{End}(\operatorname{soc} M) \simeq E$ and $M$ is not injective.

Proof. Let $R$ be the matrix algebra $\left(\begin{array}{ccc}K & 0 & 0 \\ 0 & K & 0 \\ E & E & E\end{array}\right)$. Next let $P$ be the left ideal $\left(\begin{array}{ccc}K & 0 & 0 \\ 0 & K & 0 \\ E & E & 0\end{array}\right)$, and let $Q$ be the left ideal $\left\{\left(\begin{array}{ccc}0 & 0 & 0 \\ 0 & 0 & 0 \\ a & -a & 0\end{array}\right) \mid a \in E\right\}$. Finally let $M=$ $P / Q, L_{1}=\left(\begin{array}{ccc}K & 0 & 0 \\ 0 & 0 & 0 \\ E & E & 0\end{array}\right), L_{2}=\left(\begin{array}{ccc}0 & 0 & 0 \\ 0 & K & 0 \\ E & E & 0\end{array}\right), N_{1}=L_{1} / Q$ and $N_{2}=L_{2} / Q$. Then $N_{1}$, $N_{2}$ and $N_{1} \cap N_{2}=\operatorname{soc} M$ are the unique proper submodules of $M$. Moreover $M / N_{1}$ and $M / N_{2}$ have dimension one over $K$ and we have $M / N_{1} \neq M / N_{2}$. Hence $M$ satisfies (123), and we clearly have $\operatorname{End}(\operatorname{soc} M) \simeq E$. Moreover it is easy to check that $M$ is a faithful $R$-module. Finally, let $\widetilde{P}$ be the left $R$-module $\left(\begin{array}{ccc}E & 0 & 0 \\ 0 & E & 0 \\ E & E & 0\end{array}\right)$. It remains to check that $M=P / Q$ is an essential submodule of $\widetilde{P} / Q$. To see this, take any $\widetilde{p}=\left(\begin{array}{ccc}x & 0 & 0 \\ 0 & y & 0 \\ z & t & 0\end{array}\right) \in \widetilde{P} \backslash P$. Then $\left(\begin{array}{lll}0 & 0 & 0 \\ 0 & 0 & 0 \\ 1 & 0 & 0\end{array}\right) \widetilde{p}=\left(\begin{array}{lll}0 & 0 & 0 \\ 0 & 0 & 0 \\ x & 0 & 0\end{array}\right)$ and $\left(\begin{array}{lll}0 & 0 & 0 \\ 0 & 0 & 0 \\ 0 & 1 & 0\end{array}\right) \widetilde{p}=$ $\left(\begin{array}{lll}0 & 0 & 0 \\ 0 & 0 & 0 \\ 0 & y & 0\end{array}\right)$. Consequently $R(\tilde{p}+Q) \cap P / Q \neq 0$, and so $P / Q$ is an essential submodule of $\widetilde{P} / Q$, as claimed.

Corollary 3.5. Any infinite cardinal $d$ is the dimension over $K$ of a faithful module satisfying (123).

Proof. If $E$ is an extension field of $K$ with $[E: K]=d$, then the claim follows from the proof of Proposition 3.4

As the next result shows, only two $K$-algebras occur as the endomorphism ring of a module satisfying (123) and defined over a $K$-algebra. 
Theorem 3.6. Let $M$ be a module satisfying (123) and defined over a $K$-algebra. Let $N_{1}$ and $N_{2}$ be the two maximal submodules of $M$. The following facts hold:

(i) If $N_{1} \cap N_{2} \nsucceq M / N_{i}$ for any $i$, then End $M$ is isomorphic to $K$.

(ii) If $N_{1} \cap N_{2} \simeq M / N_{i}$ for some $i$, then End $M$ is isomorphic to $K[x] /\left(x^{2}\right)$.

Proof. Let $\pi: M \longrightarrow M /\left(N_{1} \cap N_{2}\right)$ be the canonical morphism. Since $N_{1} \cap N_{2}$ is fully invariant in $M$, for any $f \in \operatorname{End} M$ there is a unique morphism $f^{\star} \in$ End $\left(M /\left(N_{1} \cap N_{2}\right)\right)$ such that $\pi \circ f=f^{\star} \circ \pi$. Let $\rho:$ End $M \rightarrow \operatorname{End}\left(M /\left(N_{1} \cap N_{2}\right)\right) \simeq$ $K \oplus K$ be the morphism sending $f$ to $f^{\star}$ for any $f$. We first note that

$$
\text { End }\left(M /\left(N_{1} \cap N_{2}\right)\right) \text { has non trivial idempotents. }
$$

We next observe that

$$
f^{2}=0 \quad \text { for every } f \in \operatorname{Ker} \rho .
$$

Since $M$ is indecomposable and idempotents lift modulo nil ideals, we conclude that

$$
\rho \text { is not surjective. }
$$

Suppose first $N_{1} \cap N_{2} \neq M / N_{i}$ for any $i$. In this case $\rho$ is injective. This remark and (3) imply that End $M$ is isomorphic to $K$. Hence (i) holds. Assume now $N_{1} \cap N_{2} \simeq M / N_{i}$ for some $i$. In this case there is some $0 \neq f \in \operatorname{Ker} \rho$. Since End $\left(M / N_{i}\right) \simeq K$, it follows that $\operatorname{Ker} \rho$ is the vector space generated by $f$. Putting (2) and (3) together, we conclude that End $M$ is isomorphic to $K[x] /\left(x^{2}\right)$. Thus also (ii) holds.

Corollary 3.7. Let $R$ be a $K$-algebra, let $M$ be a module satisfying (123), and let $E=$ End $M$. The following facts hold:

(i) $R$ is not commutative and $E$ is commutative.

(ii) $M$ is a cyclic $R$-module and $M$ is not a cyclic E-module.

Proof. (i) We know from [4, Remark 2.2] that $R$ is not commutative, while we know from Theorem 3.6 that $E$ is commutative.

(ii) Let $N_{1}$ and $N_{2}$ be the maximal submodules of $M$. Then any $m \in M \backslash\left(N_{1} \cup N_{2}\right)$ generates $M$ as an $R$-module. By Theorem 3.6 we have $\operatorname{dim} E \leq 2$. On the other hand we clearly have $\operatorname{dim} M \geq 3$. Hence $M$ is not a cyclic $E$-module.

As usually, we say that a bimodule ${ }_{A} X_{B}$ is faithfully balanced if the canonical morphisms $A \longrightarrow$ End $X_{B}$ and $B \longrightarrow \operatorname{End}_{A} X$, sending any $a \in A$ and $b \in B$ to the corresponding multiplications, are isomorphisms. 
Proposition 3.8. Let $R, M, N_{1}, N_{2}$ and $E=\operatorname{End} M$ be as in Corollary 3.7. Let $T$ be the algebra of all endomorphisms of $M$ as an $E$-module. The following facts hold:

(i) $M$ is not a faithfully balanced $R-E$ bimodule.

(ii) If $R$ is a finite dimensional algebra, then we may have $\operatorname{dim} T=\operatorname{dim} R+1$.

Proof. We first note that

$$
M \text { is not a simple } R \text {-module. }
$$

Assume first $E \simeq K$. Then $T \simeq \operatorname{End}_{K}(M)$ and $M$ is a simple $T$-module. This remark and (1) prove that $M$ is not faithfully balanced. Assume now $E=K[f]$ for some endomorphism $f$ of $M$ such that

$$
f\left(N_{1}\right)=0 \text { and } f\left(N_{2}\right)=N_{1} \cap N_{2} .
$$

Let now $B$ be a basis of $M$ of the form $B_{1} \cup B_{2} \cup B_{3}$ such that $B_{1} \cup B_{3}$ and $B_{2} \cup B_{3}$ are bases of $N_{1}$ and $N_{2}$ respectively, while $B_{3}$ is a basis of $N_{1} \cap N_{2}$. Next let $s: M \longrightarrow M$ be a $K$-linear map such that

$$
s(b)=0 \text { for any } b \in B_{1} \cup B_{3},
$$

and

$$
s(b) \in B_{1} \text { for any } b \in B_{2} .
$$

Consequently we deduce from (2), (3) and (4) that $(s \circ f)(b)=0=(f \circ s)(b)$ for any $b \in B$. It follows that $s \in T$. On the other hand we deduce from (4) that $s\left(N_{2}\right) \notin N_{2}$. Hence $s$ does not act as the multiplication by an element of $R$, and so $(i)$ holds. To prove (ii), we choose $R$ and $M$ as in [4, Example 2.4]. More precisely, let $R$ be the $K$-algebra given by the quiver $\dot{1}_{2} \stackrel{a}{\rightarrow} \bullet_{2}$ b with relations $b a=0$ and $b^{2}=0$, and let $M$ be the module $\begin{gathered}12 \\ 2\end{gathered}$. We first note that

$$
R \text { is isomorphic to the algebra of all matrices over } K \text { of the form }\left(\begin{array}{lll}
a & 0 & 0 \\
0 & b & 0 \\
c & d & b
\end{array}\right) \text {. }
$$


Consequently $E$ is isomorphic to the algebra of all matrices over $K$ of the form $\left(\begin{array}{lll}x & 0 & 0 \\ 0 & x & 0 \\ 0 & y & x\end{array}\right)$. Therefore it is easy to check that

$T$ is isomorphic to the algebra of all matrices over $K$ of the form $\left(\begin{array}{lll}a & e & 0 \\ 0 & b & 0 \\ c & d & b\end{array}\right)$

Hence, by (5) and (6), we have $\operatorname{dim} R=4$ and $\operatorname{dim} T=5$. Hence also (ii) holds.

In the next statement we collect some results on dimensions and generators.

Proposition 3.9. Let $R$ be a $K$-algebra and let $M$ be an $R$-module satisfying (123). The following facts hold:

(i) $R$ is generated by at least two elements.

(ii) $\operatorname{dim} R \geq 4$.

(iii) There is an algebra $B$, having a $B$-module satisfying (123), such that $B$ is generated by two elements and $\operatorname{dim} B=4$.

Proof. (i) This follows from the fact that $R$ is not commutative (Corollary 3.7).

(ii) We first note that $M$ is a cyclic $R$-module which is not projective (Corollary 3.7 and [4, Lemma 2.1]). Consequently either $\operatorname{dim} R=\infty$ or $3 \leq \operatorname{dim} M<$ $\operatorname{dim} R<\infty$.

(iii) Let $B$ be the $K$-algebra given by the quiver $b a=0$ and $b^{2}=0$, used to prove condition (ii) of Proposition 3.8. Next let $e_{1}$ and $e_{2}$ be the paths of length zero around 1 and 2 . Then $\left\{e_{1}, e_{2}, a, b\right\}$ is a basis of $B$, and we clearly have $e_{2}=1-e_{1}, a=(a+b) e_{1}$ and $b=(a+b) e_{2}=$ $(a+b)\left(1-e_{1}\right)$. Hence $B$ is generated by $e_{1}$ and $a+b$.

The proof of Proposition 2.1 shows that many modules of dimension 3, satisfying (123), are injective modules over suitable algebras. We finally show that also the previous $K\langle x, y\rangle$-modules of dimension 3 have this property.

Corollary 3.10. Let $B$ be the K-algebra used to prove Proposition 3.9 (iii), and let $M$ be the $K\langle x, y\rangle$-module used to prove Proposition 2.6 (ii) (resp. (iii)). Then $M$ is a faithful injective $B$-module. 
Proof. By hypothesis $M$ is described by the following picture
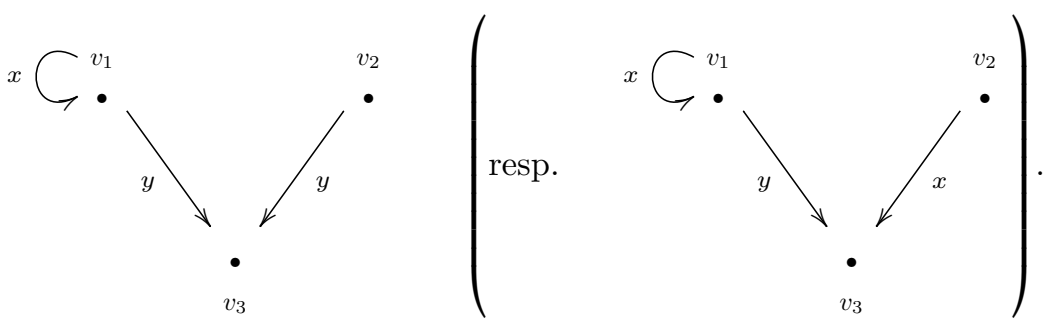

Let $f$ and $g$ be the endomorphisms of $M$ induced by $x$ and $y$. Then we clearly have $f^{2}=f, g^{2}=0, f g=0$ and $g f \neq 0$ (resp. $f^{3}=f^{2}, g^{2}=0, f g=0$ and $g f=g$ ). On the other hand, by Proposition 3.9 we have $\operatorname{dim} K\langle f, g\rangle \geq 4$. Consequently $\{1, f, g, g f\}$ (resp. $\left\{1, f, f^{2}, g\right\}$ ) is a basis of $K\langle f, g\rangle$. Moreover the $K$-linear map $B \longrightarrow K\langle f, g\rangle$ such that $e_{1} \mapsto f, e_{2} \mapsto 1-f, a \mapsto g f, b \mapsto g-g f$ (resp. $e_{1} \mapsto f^{2}, e_{2} \mapsto 1-f^{2}$, $\left.a \mapsto g, b \mapsto f-f^{2}\right)$ is an algebra isomorphism. Since $\begin{gathered}1 \\ 2\end{gathered}, \frac{2}{2}, \begin{gathered}12 \\ 2\end{gathered}, 1,2$, are the indecomposable $B$-modules, we conclude that the $B$-module $M$ is the injective module $\begin{gathered}12 \\ 2\end{gathered}$.

Corollary 3.11. Let $C$ be the $K$-algebra given by the Dynkin diagram

$$
1 \stackrel{a}{\longrightarrow} 3 \stackrel{b}{\longleftarrow} 2
$$

and let $M$ be the $K\langle x, y\rangle$-module constructed in Example 2.5. Then $M$ is a faithful injective $C$-module.

Proof. By hypothesis $M$ is described by the following picture.

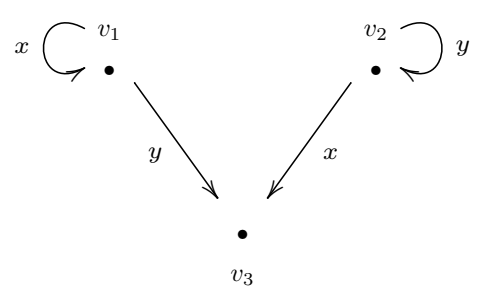

Let $f$ and $g$ be the endomorphisms of $M$ induced by $x$ and $y$. Then we clearly have $f^{3}=f^{2}, g^{3}=g^{2}, f g=f g^{2}=f-f^{2}, g f=g f^{2}=g-g^{2}$, and $f^{2} g=g^{2} f=0$. Moreover $\left\{1, f, f^{2}, g, g^{2}\right\}$ is a basis of $K\langle f, g\rangle$. As always, let $e_{i}$ be the path of length zero around the vertex $i$. Then the $K$-linear map $C \longrightarrow K\langle f, g\rangle$ such that $e_{1} \mapsto f^{2}$, $e_{2} \mapsto g^{2}, e_{3} \mapsto 1-f^{2}-g^{2}, a \mapsto g-g^{2}, b \mapsto f-f^{2}$ is an algebra isomorphism. Hence the $C$-module $M$ is the injective module $\begin{gathered}12 \\ 3\end{gathered}$. 
In all the previous examples of faithful modules $M$, satisfying (123) and defined over finite dimensional $K$-algebras $R$, we always have $\operatorname{dim} R-\operatorname{dim} M \leq 2$. To see that this is not always true, it suffices to consider the following example.

Example 3.12. There are a $K$-algebra $R$ and a faithful $R$-module $M$, satisfying (123), such that $\operatorname{dim} M=4$, End $M \simeq K$ and $\operatorname{dim} R=10$.

Construction. Let $M$ be the $K\langle x, y, z\rangle$-module described by the following picture.

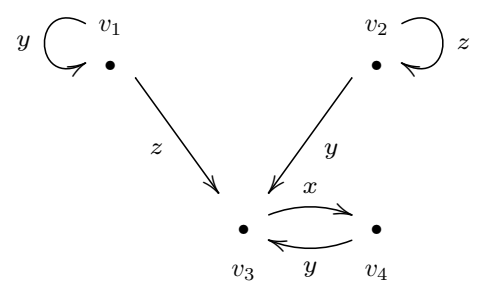

Then we deduce from Theorems 3.2 and 3.6 that $M$ is a $K\langle x, y, z\rangle$-module satisfying (123) with End $M \simeq K$ and $\operatorname{dim} M=4$. Let $f, g, h$ be the endormorphisms of $M$ induced by $x, y, z$ respectively, and let $R=K\langle f, g, h\rangle$. Next let $e=1-g^{2}-h^{2}-g f$ and $\ell=g e$. Then the linear maps $g^{2}, h^{2}, h g, g h, g f, \ell, f h, f g h, f, e$ are described by ten different matrices (with entries in $K$ ) with one entry equal to 1 and the remaining ones equal to 0 , as suggested by the following matrix

$$
\left(\begin{array}{cccc}
g^{2} & 0 & 0 & 0 \\
0 & h^{2} & 0 & 0 \\
h g & g h & g f & \ell \\
f h & f g h & f & e
\end{array}\right) .
$$

This remark and the shape of $M$ imply that $R$ is isomorphic to the matrix algebra

$$
\left(\begin{array}{llll}
K & 0 & 0 & 0 \\
0 & K & 0 & 0 \\
K & K & K & K \\
K & K & K & K
\end{array}\right)
$$

Consequently we have $\operatorname{dim} R=10$, as claimed.

As the next example shows, the dimension of a faithful module satisfying (123) and that of its endomorphism ring do not determine the dimension of the algebra.

Example 3.13. There are a $K$-algebra $A$ and a faithful $A$-module $L$, satisfying (123), such that $\operatorname{dim} L=4$, End $L \simeq K$ and $\operatorname{dim} A=9$. 
Construction. Let $L$ be the $K\langle x, y, z\rangle$-module described by the following picture.

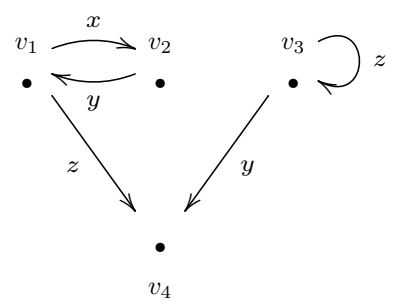

Then we have $\operatorname{dim} L=4$. Proceeding as in Theorem 3.2 we conclude that the $K\langle x, y, z\rangle$-module $L$ satisfies (123). Hence we deduce from Theorem 3.6 that End $L \simeq K$. Next let $f, g, h$ be the endomorphisms of $L$ induced by $x, y, z$ respectively, and let $A=K\langle f, g, h\rangle$. Finally let $e=1-g f-f g-h^{2}$. Then the elements $g f, g f g, f, f g, h^{2}, h g f, h g, g h, e$ are described by nine different matrices (with entries in $K$ ) with one entry equal to 1 and the remaining ones equal to 0 , as suggested by the following matrix

$$
\left(\begin{array}{cccc}
g f & g f g & 0 & 0 \\
f & f g & 0 & 0 \\
0 & 0 & h^{2} & 0 \\
h g f & h g & g h & e
\end{array}\right) .
$$

This observation and the shape of $M$ imply that $A$ is isomorphic to the matrix algebra

Hence we have $\operatorname{dim} A=9$.

$$
\left(\begin{array}{llll}
K & K & 0 & 0 \\
K & K & 0 & 0 \\
0 & 0 & K & 0 \\
K & K & K & K
\end{array}\right) .
$$

Example 3.14. There are a $K$-algebra $B$ and a faithful $B$-module $U$, satisfying (123), such that $\operatorname{dim} U=4$, End $U \simeq K[x] /\left(x^{2}\right)$ and $\operatorname{dim} B=8$.

Construction. Let $U$ be the $K\langle x, y, z\rangle$-module described by the following picture.

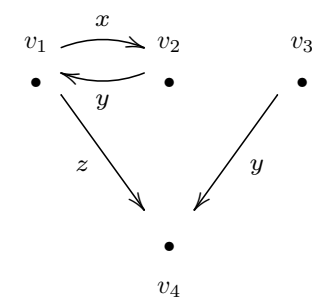

Let $f, g, h$ be the endomorphisms of $U$ induced by $x, y, z$ respectively, and let $B=$ $K\langle f, g, h\rangle$. Then $U$ is a faithful $B$-module with $\operatorname{dim} U=4$ and, proceeding as in 
Theorem 3.2 it is easy to see that the $B$-module $U$ satisfies (123). Finally, by Theorem 3.6. we have End $U \simeq K[x] /\left(x^{2}\right)$. Let $e=1-g f-f g$ and let $\ell=g-g f g$. Then the elements $g f, g f g, f, f g, e, h, h g, \ell$ are described by matrices with one or two entries equal to 1 and the remaining ones equal to 0 , as suggested by the following matrix

$$
\left(\begin{array}{cccc}
g f & g f g & 0 & 0 \\
f & f g & 0 & 0 \\
0 & 0 & e & 0 \\
h & h g & \ell & e
\end{array}\right) .
$$

This remark and the shape of $U$ imply that $B$ is the $K$-algebra of all matrices (with entries in $K$ ) of the form

$$
\left(\begin{array}{llll}
\star & \star & 0 & 0 \\
\star & \star & 0 & 0 \\
0 & 0 & t & 0 \\
\star & \star & \star & t
\end{array}\right) .
$$

Hence we have $\operatorname{dim} B=8$, as claimed.

Corollary 3.15. There exist two finite dimensional factor algebras $A$ and $B$ of $K\langle x, y, z\rangle$ and two faithful modules ${ }_{A} L$ and ${ }_{B} U$, satisfying (123), such that $\operatorname{dim} L=$ $\operatorname{dim} U, \operatorname{dim} \operatorname{soc} L=\operatorname{dim} \operatorname{soc} U, \operatorname{dim} A+\operatorname{dim} \operatorname{End} L=\operatorname{dim} B+\operatorname{dim} \operatorname{End} U$ and $\operatorname{dim} A \neq$ $\operatorname{dim} B$.

Proof. Let $A, B, L$ and $U$ be as in Examples 3.13 and 3.14 . Then we have $\operatorname{dim} L=$ $\operatorname{dim} U=4, \operatorname{dim} \operatorname{soc} L=\operatorname{dim} \operatorname{soc} U=1, \operatorname{dim} A=9, \operatorname{dim} B=8, \operatorname{dim}$ End $L=1$ and $\operatorname{dim} \operatorname{End} U=2$.

\section{References}

[1] I. Assem, D. Simson and A. Skowronski, Elements of the Representation Theory of Associative Algebras, Vol. 1, London Mathematical Society Student Texts, 65, Cambridge University Press, Cambridge, 2006.

[2] M. Auslander, I. Reiten and S. O. Smalø, Representation Theory of Artin Algebras, Cambridge Studies in Advanced Mathematics, 36, Cambridge University Press, Cambridge, 1995.

[3] J. Clark, C. Lomp, N. Vanaja and R. Wisbauer, Lifting Modules, Supplements and Projectivity in Module Theory, Frontiers in Math., Boston, Birkhäuser, 2006.

[4] G. D'Este and D. Keskin Tütüncü, Pseudo projective modules which are not quasi projective and quivers, Taiwanese J. Math., 22(5) (2018), 1083-1090. 
[5] P. A. Guil Asensio, D. Keskin Tütüncü, B. Kalebog̃az and A. K. Srivastava, Modules which are coinvariant under automorphisms of their projective covers, J. Algebra, 466 (2016), 147-152.

\section{Gabriella D'Este}

Department of Mathematics

University of Milano

Via Saldini 50

20133 Milano, Italy

e-mail: gabriella.deste@unimi.it 